engaged in formal sex work. STIs including Ureaplasma urealyticum (UU), Chlamydia trachomatis (CT), Mycoplasma hominis (MH), Trichomonas vaginalis (TV), Mycoplasma genitalium $(\mathrm{MG})$ and Neisseria gonorrhea (NG) were detected using the Seegene Anyplex qPCR panel. The cervicovaginal microbiome and cytokines were also characterized in a subset of 168 participants.

Results The prevalence of any STI was 25.1\% (95\% CI, 22.328.0\%). The most common STIs were UU (13.2\%) and $\mathrm{MH}$ (11.3\%), followed by CT (6.6\%), TV (3.2\%), NG (0.3\%), and MG (0.5\%). U. urealyticum was more prevalent among sex workers compared to non-sex workers (19.7 vs $10.4 \%$, $\mathrm{p}=0.001)$. Number of partners positively correlated with UU $(\mathrm{P}=0.004)$, while duration of sexual activity was positively correlated with $\mathrm{MH}(\mathrm{P}<0.001)$ and UU $(\mathrm{P}=0.023)$. Recent history of STI treatment did not correlate with any STI. $\mathrm{MH}$ was most associated with symptoms, including vaginal discharge, itching, and painful urination $(\mathrm{P}<0.001)$. All STIs except CT were positively correlated with pro-inflammatory cytokines. UU and $\mathrm{MH}$ were correlated with vaginal microbiota alpha diversity $(\mathrm{P}<0.001)$.

Conclusion $U$. urealyticum and $M$. hominis were the most prevalent STIs among young women from Mombasa, Kenya, and these organisms were associated with sexual exposure and an inflammatory mucosal milieu that has been linked to increased HIV acquisition.

Disclosure No significant relationships.

\section{P339 FACTORS OF RISKY SEXUAL BEHAVIOR AMONG LONG- HAUL TRUCKERS IN A SOUTHERN NIGERIAN TOWN}

\footnotetext{
${ }^{1}$ Paul Okojie*, ${ }^{2}$ Kingsley Okafor, ${ }^{3}$ Omoyemen Bello, ${ }^{3}$ Esohe Ogboghodo, ${ }^{3}$ Abimbola Adesanya. 'Liberty University, Public and Community Health, Lynchburg, USA; ${ }^{2}$ Health Research and Policy Development Foundation, Abuja, Nigeria; ${ }^{3}$ University of Benin, Community Health, Benin City, Nigeria
}

10.1136/sextrans-2019-sti.447

Background Long-haul drivers play a role in the spread of sexually transmitted infections (STI) globally. Truck drivers engage in frequent travel away from home. They are exposed to risky sex with commercial sex workers who are a reservoir of HIV and other STIs. The study aimed to identify factors associated with commercial sexual exposure among long-haul truckers in a southern Nigerian town.

Methods A sample of 300 long-haul truckers from a highway park in Ahor, southern Nigeria was recruited in a cross-sectional study. An interviewer-administered questionnaire was used to collect socio-demographic and sexual risk behavior data. Data were analyzed with SPSS version 20 software. Chisquare test, Odds ratio, and confidence intervals were used to find the association between condom use, STI history, HIV status, duration of a trip, number of sexual partners, and truckers' exposure to commercial sex workers.

Results While $42.0 \%$ of the truckers used condoms, $58.0 \%$ were non-users. Reasons for non-condom use were commitment to partner (72.0\%), lack of pleasure (21.0\%), and beliefs (7.0\%). Fifty percent of respondents reported two or less sexual contacts while a third of them reported 12 sexual contacts in 6 months. Overall, $59.0 \%$ of respondents had a history of multiple sexual partners. Factors associated with truckers' exposure to a commercial sex worker were use of psychoactive substance (OR 2.5 (1.5-4.4), $p=0.00$; condom use (OR 16.3 (8.0-32.5), $p=0.00$; previous sexually transmitted infection (OR $2.5(1.2-5.1), p=0.01$. The odds of single-sex partner exposure to a commercial sex worker were $10 \%$ less than the odds in multiple sex partner truckers, $p=0.00$.

Conclusion This study shows that psychoactive substances and condoms may be fueling exposure to commercial sex among truck drivers. Intervention programs should emphasize a single partner relationship, target substance users and highlight the potential impact of previous sexually transmitted infections on the risk of acquiring an HIV infection.

Disclosure No significant relationships.

\section{P340 TRENDS IN HIGH-RISK SEXUAL RISK BEHAVIORS IN THE UNITED STATES, 2002-2015}

Jami Leichliter, Patricia Dittus*, Sevgi Aral. CDC, Atlanta, USA

\subsection{6/sextrans-2019-sti.448}

Background Reportable STIs have largely been increasing in the United States; yet, few national-level studies have examined potential reasons for the increase. We used data from the 2002 and 2011-15 National Survey of Family Growth to examine trends in high-risk sexual behavior among key subpopulations with disproportionate STI rates.

Methods The subpopulations we examined included sexually active: men who have sex with men (MSM) and adolescent, young adult, Hispanic, and non-Hispanic black women who had sex with men (WSM) and men who had sex with women (MSW). High-risk sexual behavior (HRSB) was a composite variable (yes, no) that included endorsement of any of the following: giving or receiving money or drugs for sex or having a partner who was bisexual, non-monogamous, HIV-positive, or who injected drugs. We used weighted data to examine the prevalence of HRSB over time for each subpopulation and conduct logistic regressions adjusting for race/ethnicity, age, marital status, poverty and education.

Results HRSB either did not change or declined over time. Overall, among WSM, reported HRSB significantly declined over time (15.1\% in 2002 and 12.4\% in 2011-15). Among men, we also found significant declines in reported HRSB for MSW (15.4\% in 2002 and $12.2 \%$ in 2011-15) and MSM $(25.9 \%$ in 2002 and $13.1 \%$ in $2011-15)$. In subgroup analyses, there were more declines among WSM than MSW or MSM. However, in adjusted analyses, we found significant declines in reported HRSB, comparing 2002 to 2011-15, among non-Hispanic black $(\mathrm{aOR}=0.78,0.65-0.95)$ and adolescent $(\mathrm{aOR}=0.64,0.50-0.95) \quad \mathrm{WSM} ;$ Hispanic $(\mathrm{aOR}=0.49$, $0.35-0.67)$ and adolescent $(\mathrm{aOR}=0.57,0.41-0.79) \mathrm{MSW}$; and all MSM $(\mathrm{aOR}=0.42,0.24-0.75)$.

Conclusion While STIs are increasing, HRSB was steady or declined among key subpopulations with disproportionate STI rates. Results from adjusted analyses suggest these findings are not the result of changing population demographics. Further research is needed to explain the STI increases.

Disclosure No significant relationships. 